\title{
A RICH CLUSTER REDSHIFT SURVEY FOR LARGE-SCALE STRUCTURE STUDIES
}

\author{
D. BATUSKI ${ }^{1}$, K. SLINGLEND $^{1}$, J. M. HILL ${ }^{2}$, S. HAASE ${ }^{1}$, \\ C. MILLER ${ }^{1}$ AND K. MICHAUD ${ }^{1}$ \\ ${ }^{1}$ Department of Physics and Astronomy, University of Maine \\ ${ }^{2}$ Steward Observatory, University of Arizona
}

\section{Introduction}

We have used the MX multifiber spectrometer on the Steward $2.3 \mathrm{~m}$ measuring redshifts of up to 25 galaxies in each of about 90 Abell cluster fields with richness class $R \geq 1$ and $\operatorname{mag}_{10} \leq 16.8$ (estimated $z \leq 0.12$ ) and no more than one previously-measured redshift. This work has resulted in a deeper, more complete sample for two-point correlation and other studies of large-scale structure. To date, we have collected such data for 110 clusters (a few not in our sample). For most, we have seven or more cluster members with redshifts, enough to add significantly to the available sample of cluster velocity dispersions for other studies of cluster properties.

\section{Instrumentation and Observations}

The MX Spectrometer velocity data for this survey is a multi-aperture fiber optic device designed and built by John M. Hill of Steward Observatory. Thirty-two fibers, each on a computer-controlled positioning probe, are used to collect galaxy spectra while another 24 fibers collect background sky spectra. The spectrograph utilizes a 300 groove $/ \mathrm{mm}$ grating tilted to provide a wavelength range of $380-670 \mathrm{~nm}$ with a resolution of $1.1 \mathrm{~nm}(0.37$ $\mathrm{nm} /$ pixel). The spectra were collected on a Texas Instruments $800 \times 800$ CCD chip and, in recent observations, on a Loral $800 \times 1200$ CCD.

The spectroscopic observations took place from 1990 to 1996. One hour integrations are typically required for each cluster. Each integration yields up to 32 quality spectra down to a limiting magnitude of $\sim 18$. The spectral coverage is $\sim 300 \mathrm{~nm}$ centered at $\sim 530 \mathrm{~nm}$. Before each cluster exposure, 
comparison lamps were obtained for each fiber for later use in wavelength calibration. Twenty-four sky fibers, positioned next to the galaxy fibers on 24 of the 32 probes, collected simultaneous sky spectra for subsequent sky subtraction.

\section{Data Reduction}

Once the data were collected, reduction was performed using the NOAO IRAF package. The images were bias-subtracted and flat-field corrected. Individual spectra are located on each image and read into one-dimensional files. The spectra were wavelength-calibrated and the sky spectra that were collected by MX during the integration on the clusters were then averaged and subtracted from each of the galaxy spectra.

The last step in the reduction is the determination of the actual galaxy redshifts by cross-correlation. Nineteen spectra of nearby galaxies of known redshift are cross-correlated with each galaxy spectrum. The resulting average is our best estimate of the galaxy's redshift. We accepted a redshift if it met criteria based on the height of the cross-correlation peak, the number of template spectra that correlated well with the galaxy spectrum, and the dispersion among the estimates of the galaxy's redshift after the spectrum passed a visual inspection.

\section{Results}

Thus far, 110 clusters have been observed in this program for $99 \%$ completion to $m_{10}=16.8$. For most of the target clusters, 10 or more redshifts per cluster field were obtained, so that average velocities, velocity dispersions, and projection effects can be evaluated accurately. This means that we now have a much-enlarged sample (from 104 for the $D \leq 4$ sample of Bahcall and Soniera (1983) to 242 now) of $R \geq 1$ Abell clusters with at least two measured redshifts per cluster. Combining this sample with the recently completed survey of ACO clusters by den Hartog (1995, PhD Thesis, Leiden University), we have not only been able to calculate a refined two-point correlation function for the northern clusters, but also one for a sample covering the whole unobscured sky. For the northern cluster case, we obtain a power law two-point spatial correlation function with $\gamma=-2.26 \pm 0.14$ and $r_{0}=21.9 \pm 1.5 \mathrm{Mpc}$. For the all-sky case, we found $\gamma=-2.53 \pm 0.15$ and $r_{0}=21.0 \pm 1.5 \mathrm{Mpc}$.

\section{References}

Bahcall, N. and Soneira, R.M. 1983 Astrophys.J, 270, 20. 